\title{
Models for Comparing Athletic Performances
}

\author{
September 20, 1997
}

H. J. Grubb ${ }^{1}$

Department of Applied Statistics, The University of Reading

\section{Summary}

We explore models for performance in athletic (running) events at a range of different distances and suggest a parametric form which can be useful for characterising the change in performance with distance. This model is fitted to 1996 world records for various distances. Performance is expressed as the average speed for each distance, which is a more natural variable than time, when considering different distances, with widely varying times. We apply the model to compare performances by the same athlete at different distances, and so to determine an athlete's strengths, or to assess the effect of training. This requires that we also examine times near to the world records in order to characterise this change in performance. Another application of our model is to jointly describe the decrease in several world records over a number of years and so to predict lower bounds on these records. We demonstrate this using some simple parametric forms with asymptotes.

Keywords: World records in running; parametric models; non-linear fitting; prediction

\section{Introduction}

We consider the problem of comparing performances in athletic events at a range of distances - Figure 1 shows the men's and women's world record times, at the end of the 1996 season, for distances from $100 \mathrm{~m}$ to the marathon, plotted against the distance. We wish to analyse

\footnotetext{
${ }^{1}$ Address for correspondence: Department of Applied Statistics, The University of Reading, PO Box 240 , READING RG6 6FN
}

e-mail: H.J.Grubb@reading.ac.uk 
and model the change in these times with distance - notice that although the record times increase apparently almost linearly with distance - see the fitted straight lines on Figure 1 for illustration - this clearly cannot be the case, since the average speed for each distance must be decreasing.

\section{Figure 1 about here}

Several previous studies have attempted to model performance, using models of varying complexity and physiological interpretability - e.g. Henry (1955), Purdy (1973), Francis (1943) and Riegel (1981). Our work is concerned with fitting the observed patterns accurately and using the parameters, or the fitted values in subsequent analyses. In this paper, we examine models previously fitted to the world record data and consider refinements of them, which better represent the features observed. Having developed a suitable model, we then use this in two ways: firstly we can normalise athletic performances with respect to the fitted values, for instance to determine which current world record is particularly outstanding, or to compare performances by an individual athlete at different distances, without the influence of exceptional world records. For this comparison, we also need some indication of how performances decrease away from the world record - the world top 20 rankings for each event are used for this. Secondly we can fit a set of such models to world records over a sequence of years and examine the change in parameter estimates through time. These can then be used to explore lower bounds on parameters and hence on world record performances. This second application has been studied by, among others, Chatterjee and Chatterjee (1982) both for individual distances and for a joint additive model for several distances. Also Blest (1996) considered several distances in one model, and we extend this work using more flexible models for performance on a natural scale, which gives us greater precision in estimation.

In $\S 2$ we explore and fit models to the world record data. We then use these models in $\S 3$ to compare performance at different distances for an individual athlete, and in $\S 4$ to address the issue of long-term trends and limits to performance. 


\section{Models}

\subsection{Some existing models}

The world record times are often viewed on a log scale, since the range of distances included is quite large - see Figure 2.

\section{Figure 2 about here}

The difference between men and women on this scale is visually smaller - it is now a ratio (from 1.10 at $200 \mathrm{~m}$ to 1.13 at the marathon). Models are often fitted on this scale - e.g. Blest (1996) and Riegel (1981) - the linear fit shown in Figure 2, being equivalent to:

$$
\begin{aligned}
\log \left(t_{i}\right) & =a+b \log \left(d_{i}\right) \\
\text { or } t_{i} & =\exp (a) d_{i}^{b}
\end{aligned}
$$

for parameters $a$ and $b$, where $t_{i}$ (secs) is the current world record time for the $i$ th distance $d_{i}$ (metres). We fit this model using simple linear regression to minimise the sum of squared residuals from (1), i.e. log time, or relative residual, rather than the residuals from (2), in time, which would favour the longer distances. We have included the six events: 400m, $800 \mathrm{~m}, 1500 \mathrm{~m}, 5000 \mathrm{~m}, 10000 \mathrm{~m}$ and marathon $(42165 \mathrm{~m})$, although the figure also shows the $100 \mathrm{~m}, 200 \mathrm{~m}$ and some intermediate distances, which either are not run so frequently, or else are similar to one of our six (e.g. the mile). We exclude the sprint events from the analysis as these are run essentially anaerobically and are dependent upon the start, as we discuss later. The estimated parameters from these fits are given in Table 1 below (estimated standard errors in brackets), together with the residual sum of squares in both $\log (t)$ and speed, $v$ (which we will refer to later).

\section{Table 1 about here}

These suggest that the time taken increases more than linearly $(\hat{b}>1)$ with distance, as we expect, and this increase is very similar for both men and women. The parameter $a$ is a fixed offset for women's performances (which equates to a ratio of times of about 1.1, as we 
have seen).

Robinson and Tawn (1995) consider a simple model $t_{3000 m}=2 c t_{1500 m}$, for comparing only the $1500 \mathrm{~m}$ and $3000 \mathrm{~m}$ times, with $\hat{c}=1.08$. Model (1) above implies that $t_{3000 \mathrm{~m}}=2^{b} t_{1500 \mathrm{~m}}$, $2^{\hat{b}}=2.14$, which is very similar.

Rather than develop refinements to the above models, we will consider a more natural scale for comparison of different events.

\section{$2.2 \quad$ Average speed}

To see the patterns in performance at different distances more clearly, we consider the average speed $v=d / t(\mathrm{~m} / \mathrm{s})$ for each event. This is the natural scale for comparing distances with widely differing times, but relatively similar speeds. We note that Keller (1974) derived equations for the optimal instantaneous speed during a race, based upon considerations of energy use by the athlete and concluded that for distances greater than about $800 \mathrm{~m}$ the race should be run at a steady pace throughout, hence justifying the use of average speed. This again distinguishes the sprint events with a significant proportion of the race time spent accelerating.

Figure 3 shows the speed of the world records against the log of their distance, which shows clearly that the longer events are run at a progressively slower speed. This is the change in performance which we are seeking and the slowing with distance is easier to see on this scale. A horizontal line in this plot would represent a constant speed for all distances, or a perfectly linear increase in Figure 1, which would not be reasonable. A straight line $\left(v_{i}=a+b d_{i}\right.$ or $\left.v_{i}=a+b \log \left(d_{i}\right)\right)$ would also not be a particularly good fit to this relationship. We show model (1) above transformed in terms of speed as the solid lines in Figure 3 - see $\S 2.2 .2$.

\section{Figure 3 about here}

Francis (1943), Henry (1955) and later Purdy (1973) modelled the changes in speed 
with distance - the so-called 'running curve'. We will use Francis' model in $§ 2.3$. Henry's model involved several exponential terms to represent various physiological processes at the different distances and while describing the patterns in Figure 3 quite accurately, particularly in the transitions from sprint to middle to long distance events as each term of the model takes effect, it is highly-parameterised and difficult to fit. Purdy (1973) considered many models for the 'running curve' and re-estimated Henry's physiological model using non-linear least-squares.

\subsubsection{Sprints}

One point to note is that the $100 \mathrm{~m}$ and $200 \mathrm{~m}$ events show quite different behaviour, as the speed 'curve' flattens off for these - maximum (average) speed appears to be achieved around $150 \mathrm{~m}$, so in fact for the men's records the $200 \mathrm{~m}$ is run at a higher average speed than the $100 \mathrm{~m}$, although the same is not yet true of the women's records. To capture this turning point, we would need quite a highly parameterised model. However, we do not have many data points available, so instead we follow Francis (1943) and usually ignore the two sprint events, suggesting that they are sufficiently different (being run primarily anaerobically) and dependent upon the start and acceleration phase of the race to require a different type of modelling.

\subsubsection{Models in terms of speed}

The fit shown by the solid lines in Figure 3 is the rearrangement of models (1) and (2) above in terms of speed:

$$
\begin{aligned}
\log \left(v_{i}\right) & =-a+(1-b) \log \left(d_{i}\right) \\
\text { or } v_{i} & =\exp (-a) d_{i}^{1-b}
\end{aligned}
$$

We can estimate model (4) in terms of $(\log )$ speed, although the fitted values, residuals and (transformed) parameter estimates do not change a great deal. 
We can see from Figure 3 that this is a poor approximation to the change in speed with distance that we observe, even though in Figures 1 and 2 the fits to time and $\log ($ time $)$ appear to be reasonable, as these are dominated by the nearly linear increase in time with distance. We seek a more flexible model for this speed decrease.

\subsection{A more flexible model}

Francis (1943) proposed the following simple model for average speed as a function of event distance (for distances above $200 \mathrm{~m}$ ):

$$
v_{i}=\frac{A}{\log \left(d_{i}\right)-B}+C
$$

for parameters $A, B$ and $C: C$ is the speed at very long distances, $\exp (B)$ is an asymptote, which might be interpreted as the distance at which maximum speed is attained, and $A$ measures the decrease in speed with (transformed) distance. To fit this model, we use a non-linear optimisation routine to minimise the sum of squared residuals in speed. For the data above, we obtain the estimated parameters shown in Table 2.

\section{Table 2 about here}

These parameters suggest that women reach their maximum speed at a longer distance than men - from $\exp (\hat{B})=67.4 \mathrm{~m}$ vs $50.07 \mathrm{~m}$, although this term is estimated on distances much greater than this; that they run slower at longer distances $(\hat{C})$, but that their decrease in speed with distance $(\hat{A})$ is less. Model (5) gives us the dashed curves shown in Figure 3 . We note that the fit is much better than that for the power law model (1) in both RSS $(v)$ and $\operatorname{RSS}(\log (t))$,

To obtain standard errors of these estimates we might use for example the jackknife method. However, with such a small sample, this is unlikely to be reliable, so instead we simplify the model, while retaining it's flexibility. 


\subsection{Linearisation}

We see from model (5) that a key element is the inverse of the log transform of (scaled) distance. The asymptote term $(B)$ introduces non-linearity to the model, which is hard to justify on such a small sample size. Also, the asymptote means that this model cannot represent sprint events. Instead of the $\log$ transform, we could consider fitting some other (power) transform of distance and trying to reformulate the model in a linear form, to make estimation easier (for instance, model (4) results in a power of $1-\hat{b}=-0.1$, instead of $\log$ ). An example we considered is based upon a shifted power transform (or 'started power', e.g. Mosteller and Tukey (1977)):

$$
v_{i}=A(d-B)^{\lambda}+C
$$

for some parameters $A, B$, and $C$ and a (negative) power $\lambda . C$ is again speed at long distances, $B$ is the distance at which maximum speed is attained and $A$ measures the decrease in speed with transformed distance. We fit this model to the world record data and display the results in Table 3 below.

\section{Table 3 about here}

We fixed the location parameter $B$ after finding that it did not vary a great deal between fits on both the 8 th best (below) and the world record data, for various years and for men and women. Keller (1974) estimated a distance of $291 \mathrm{~m}$ at which the race changes from a sprint to a long-distance race, using different energy sources, so our estimate of $250 \mathrm{~m}$ seems reasonable. Similarly we fixed the power $\lambda$, which is equivalent to choosing the log transform in model (5). The full parameterisation of model (6) is ill-determined, so by constraining these two parameters, $B$ and $\lambda$, based upon our empirical observations we can then better determine the other two ( $A$ and $C$ ) and so interpret changes in these - see section 4 .

Having fixed the parameters $B$ and $\lambda$ we can also estimate model (6) by ordinary least squares to obtain standard errors for the estimated parameters - shown in Table 3 above. 


\section{$2.5 \quad 8$ th best times}

World records are by their nature extremes, so that we may be a little cautious about fitting to these - we observe considerable scatter around the curves in Figure 3. We could consider adapting our procedures to account for the extreme value behaviour, although we have relatively little information from the small sample size. However, we also have available, the current top 20 times for each event, which should give a more representative pattern of performance. Figure 4 plots the speeds of the 8 th best times with some fitted curves. We have chosen the 8 th best as a median summary, since some of the top 20 times included results by the same athlete, so excluding these gives us about 16 results for each event. We see that these speeds exhibit a much smoother decrease with (log) distance than the records (also shown).

\section{Figure 4 about here}

We can refit our model (5) to this to obtain some small changes in the estimated parameters. Similarly we refit model $(3)($ to $\log (v))$, giving the residual sums of squares shown in Table 4 below.

\section{Table 4 about here}

We also estimate model (6) for these data, using ordinary least squares - see Table 5.

\section{Table 5 about here}

This model now suggests that men run about $1.0 \mathrm{~m} / \mathrm{s}$ faster than women at shorter distances, reducing to $0.6 \mathrm{~m} / \mathrm{s}$ faster at longer distances. Model $(6)$ is the best-fitting of the models we have considered.

\subsection{Estimated times}

We can examine the estimated times from this model, both for distances within the fit and for intermediate distances which we have not included, see Table 6 .

\section{Table 6 about here}


For the women, while the $1000 \mathrm{~m}$ and $5000 \mathrm{~m}$ times are marginally slower than predicted from the other records, the $3000 \mathrm{~m}$ and particularly the $10000 \mathrm{~m}$ times are faster than we would expect. Both of these are held by China's Wang Junxia - see Robinson and Tawn (1995) for an examination of the $3000 \mathrm{~m}$ record. For the men, the $1000 \mathrm{~m}$ and $3000 \mathrm{~m}$ times seem reasonable, but the $5000 \mathrm{~m}$ and the $10000 \mathrm{~m}$ time are faster than we would predict. The $5000 \mathrm{~m}$ record is held by Ethiopia's Haile Gebresalassie and the $10000 \mathrm{~m}$ by Morocco's Salah Hissou. Before this was broken, Gebresalassie's previous time (26:43.53) was often considered to be one of the outstanding world records.

We note that the men's marathon is slightly under-estimated - this distance may be approaching the limit of the model validity, in particular, Henry's (1955) physiological model shows a distinct further decrease in speed around the $32 \mathrm{~km}$ point, which we are unable to capture with fewer parameters. Also, the demands of the marathon means that it will not be attempted as often as the other distances and it is the only event run on the road, so we might not expect to fit this well, although the women's fit is remarkably good.

\subsubsection{Ultra distances}

We might also consider the long-distance speed term $C$. If we interpret this as the speed an athlete might achieve when running very long distances, then we can consider, for instance the $100 \mathrm{~km}$ world records. These are currently run at about $4.42 \mathrm{~m} / \mathrm{s}$ for men $(6: 16: 41)$, and $3.96 \mathrm{~m} / \mathrm{s}$ for women $(7: 00: 47)$, which compare reasonably well with our parameter estimates

- men: $\hat{C}=4.66$, women: $\hat{C}=4.00$, in particular the estimates are still higher. Predicting from our model to these distances gives poor estimates, since this is considerably outside the range of fitting. 


\section{Comparison of performances}

An initial motivation for studying the world record data and modelling the decrease in speed with distance was to allow us to compare performances by a single athlete at a range of distances - i.e. to obtain an objective measure of performance, which is independent of the distance run. Then, for instance a $5 \mathrm{~km}$ race could be used as a predictor for a $10 \mathrm{~km}$ one or the effect of training could be seen in improved performances, not necessarily all at the

same distance. Such measures have been considered in the past e.g. Purdy (1973) derived a scoring system based upon changes to the 'running curve', while Riegel (1981) considered percentage of the world record speed as a measure of performance. We use the world top 20 data (16 individuals) to suggest a suitable comparison.

\subsection{Top 16 times}

To characterise changes in performance at each distance we consider the inter-quartile range of the top 16 times, or the difference in speed between the 4 th and 12 th times. Figure 5 shows this difference in speed, for both men and women, which shows a decrease with distance, and hence with speed - the $200 \mathrm{~m}, 400 \mathrm{~m}$ and $1500 \mathrm{~m}$ have notably large spreads, due to clusters of very fast results, probably at major championships. Instead we consider the ratio of speeds (Figure 6), which now appears more uniform.

Figure 5 about here

Figure 6 about here

\subsection{Ratio of speed}

This leads us to suggest that performances decline away from the world record approximately in a constant ratio of speed. This is based on arguably little data - the top 20 times are only available for major championship distances, although it seems reasonable and agrees with 
Riegel (1981). We then normalise results as a proportion of the actual or estimated 8th best time, avoiding problems of comparing against a particularly exceptional world record.

\subsection{An example comparison}

In practice then we compare an individual athlete's results as follows: a $10 \mathrm{~km}$ time of $2029 \mathrm{~s}$ (33:49) is $91.9 \%$ of the world 8 th best speed, while a $5 \mathrm{~km}$ time of $966 \mathrm{~s}(16: 06)$ is $91.6 \%$ of the corresponding world 8th best speed. Riegel (1981) suggests that many athletes may run at the same percentage of the world record (or in our case 8th best) speed over many distances. This seems unlikely as different athletes will have different strengths, while the world records are achieved by the best athlete at each distance - for example the same athlete above has run $121.4 \mathrm{~s}$ for $800 \mathrm{~m}$, which is $95 \%$ of the 8 th best speed, and he is known to be stronger at shorter distances. Therefore to compare different performances, we need not only normalise with respect to the world class results at each distance, but also to an athlete's current personal best at each distance - this might lead us to estimate a personal performance curve against which results can be compared.

\section{Long-term trends in records}

A further motivation for considering several different distances jointly is when measuring trends or seeking lower bounds on athletic performance. Any such study may be more powerful if applied to a range of distances than to a single one. We note however that it is likely that development of performance at different distances may not have been uniform through the years. Also, this analysis assumes that each record used is representative of the same level of performance. This may be reasonable for records at regularly contested distances, however for example, championship performances would not be suitable as these are affected by race tactics and can be relatively slow. 


\subsection{Previous work}

Such a study of long-term lower bounds on performance was presented by Blest (1996), using model (1) above. To consider change through time, this model was fitted to each Olympic year individually, then simplified to have only one intercept term $a$ for all years. The change in the parameter $b_{y}$ through the years was then modelled using various parametric forms with asymptotes, to represent a theoretical lower bound on the world record times. Note that the parameter $b$ in model (1) is interpreted as the rate of decrease in speed with distance run, so that change in this parameter should really be thought of as equalisation of average speed between different distances, rather than an overall increase in performance through the years. We display variation in the estimated parameters $a$ and $b$ together with estimated $95 \%$ confidence intervals for these for the 19 Olympic years since 1912 in Figures 7 and 8 (joint confidence intervals for the two parameters, estimates of which are correlated, are slightly wider - the marginal ones shown corresponding to approximately $90 \%$ joint coverage).

\section{Figure 7 about here}

\section{Figure 8 about here}

We also note that the residual correction used by Blest (1996) to correct for underestimation of the existing $200 \mathrm{~m}, 400 \mathrm{~m}$ and marathon records is an approximate correction for the curvature we see in Figure 3 and that there are similar residual problems at the other events.

\subsection{Trends in parameters}

To use our model (6) for this study, we also re-estimate it for each Olympic year. We consider only the men's data as the women's records do not go back beyond 1972 for most distances. Figures 9 and 10 show the variation in the estimated parameters, $A_{y}$ and $C_{y}$, together with estimated, marginal 95\% confidence intervals around these - note that these intervals are much narrower in relation to the changes in the estimates for this model, i.e. the parameters 
are determined more precisely, particularly $C_{y}$ (joint confidence intervals, as for model (6) above, are slightly wider, but the conclusion remains the same).

\section{Figure 9 about here}

Figure 10 about here

We refer to Blest (1996) for a discussion of possible parametric forms for the trend in these parameters - we fit two of these models to the observed trend in $C_{y}$. The first is the extended Chapman-Richards form - shown as the thick line on Figure 10:

$$
C_{y}=\delta-\alpha(1-\exp (-\beta y))^{\gamma}
$$

Where $y=($ year -1908$) / 4$, with $\hat{\delta}=3.71, \hat{\alpha}=-1.44, \hat{\beta}=7.07, \hat{\gamma}=1.81$ (fitted by non-linear least-squares) which gives a limit of $\hat{C}_{\infty}=\hat{\delta}-\hat{\alpha}=5.15 \mathrm{~m} / \mathrm{s}$; We also fit the anti-symmetric exponential model:

$$
\begin{aligned}
& C_{y}=\delta+\alpha \exp (-\beta(y-\gamma)) \quad y \geq \gamma \\
& C_{y}=\delta+\alpha(2-\exp (\beta(y-\gamma))) \quad y<\gamma
\end{aligned}
$$

with $\hat{\delta}=4.96, \hat{\alpha}=-0.78, \hat{\beta}=8.11, \hat{\gamma}=11.17$, with a limit of $\hat{C}_{\infty}=\hat{\delta}=4.96 \mathrm{~m} / \mathrm{s}$. The parameter $\gamma$ corresponds to a slowing in the rate of change from about 1953 onwards, which reduces the final limit.

\subsection{Lower Bounds}

Using both of these models, we then compute predicted lower bounds on times for each distance from model (6) by setting $C=\hat{C}_{\infty}$. The results are shown in Table 7 .

\section{Table 7 about here}

Clearly these results are dependent upon the parametric assumption we make - in particular equations (7) and (8) include a strong slowing in the rate of change after 1953 . However the results do suggest that the $10000 \mathrm{~m}$ record is perhaps not so extreme as is sometimes thought. 
While we must be cautious in predicting limits to performance, due to changes in training, diet and race preparation - see for example Masood (1996) - we can for instance compare the percentage of the predicted limits for different records to identify those which may perhaps be broken more easily. For example, Sebastian Coe's $800 \mathrm{~m}$ mark has stood since 1981 and still appears to be comparable to many of the other more recent records. This event is very difficult, requiring both sprinting strength and middle-distance stamina, suggesting that this record is all the more remarkable. The marathon mark also appears weak, although this is a very demanding event, with less attempts at world class level and is also at the limit of the validity of our model, so we would not necessarily forecast that this will be broken by a substantial margin in the near future.

\section{Conclusions}

We have characterised the decrease in average speed with distance as a natural and useful measure of performance for athletic events. We can describe this decrease with various parametric models. We demonstrated that a simple shifted power transformation of distance captures the observed shape of this decrease, which some common models do not. We can use the estimated curve to compare an individual athlete's performances at different distances, by computing the fraction of the fitted speed which was achieved. Also, if we assume that the shape of this curve has been constant through the years, which seems reasonable when we estimate it for each Olympic year, then we can consider the observed trend in parameters of these models and suggest limits for these and hence for the world record times. The form of our model gives apparently more precision in this estimation.

\section{Acknowledgements}

We gratefully acknowledge Dr James Street for supplying his detailed racing and training data for analysis and for stimulating discussions. Some preliminary work on this was carried 
out by Matthew Biddle and Rachel Darby-Dowman as part of their undergraduate projects at The University of Reading. Two referees provided helpful comments.

\section{References}

Blest, D.C. (1996) Lower bounds for athletic performance. The Statistician, 45, 243-253.

Chatterjee, S. and Chatterjee, S. (1982) New lamps for old: an exploratory analysis of running times in Olympic games. Applied Statistics, 31, 14-22.

Francis, A.W. (1943) Running records. Science, 98, 315-316.

Henry,F.M. (1955) Prediction of world records in running sixty yards to twenty-six miles. Research Quarterly, 26 147-158.

Keller, J.B. (1974) Optimal velocity in a race. American Mathematics Monthly, 81, 474-480.

Masood, E. (1996) Swifter, higher, stronger: pushing the envelope of performance. Nature, $382,12-16$.

Mosteller, F. and Tukey, J.W. (1977) Data Analysis and Regression. Reading MA: AddisonWesley.

Purdy, J.G. (1973) Least squares model for the running curve. Research Quarterly, 45, $224-237$.

Riegel, P.S. (1981) Athletic Records and Human Endurance. American Scientist, 69, 285290.

Robinson, M.E. and Tawn, J.A. (1995) Statistics for exceptional athletics records. Applied Statistics, 44, 499-511. 


\section{Tables}

\begin{tabular}{|r|c|c|c|c|}
\hline & $\hat{a}$ & $\hat{b}$ & $\operatorname{RSS}(\log (t))$ & $\operatorname{RSS}(v)$ \\
\hline men & $-2.75(0.027)$ & $1.100(0.0004)$ & 0.0092 & 0.588 \\
women & $-2.66(0.032)$ & $1.101(0.0005)$ & 0.0133 & 0.656 \\
\hline
\end{tabular}

Table 1: Parameters of model (1)

\begin{tabular}{|r|c|c|c|c|c|}
\hline & $\hat{A}$ & $\hat{B}$ & $\hat{C}$ & $\operatorname{RSS}(\log (t))$ & $\operatorname{RSS}(v)$ \\
\hline men & 10.43 & 3.91 & 4.19 & 0.0010 & 0.0404 \\
women & 7.99 & 4.21 & 3.90 & 0.0012 & 0.0376 \\
\hline
\end{tabular}

Table 2: Parameters of model (5)

\begin{tabular}{|r|c|c|c|c|c|c|}
\hline & $\hat{A}$ & $\hat{B}$ & $\hat{C}$ & $\hat{\lambda}$ & $\operatorname{RSS}(\log (t))$ & $\operatorname{RSS}(v)$ \\
\hline men & $17.29(0.316)$ & $250^{*}$ & $4.69(0.049)$ & $-0.267^{+}$ & 0.00044 & 0.0168 \\
women & $16.15(0.444)$ & $250^{*}$ & $4.12(0.070)$ & $-0.267^{+}$ & 0.00097 & 0.0331 \\
\hline
\end{tabular}

Table 3: Parameters of model (6) (world records)

\begin{tabular}{|c|c|c|c|}
\hline Mod & & $\operatorname{RSS}(\log (t))$ & $\operatorname{RSS}(v)$ \\
\hline \multirow[t]{2}{*}{ (3) } & men & 0.0161 & 0.979 \\
\hline & women & 0.0203 & 0.973 \\
\hline \multirow[t]{2}{*}{$(5)$} & men & 0.00044 & 0.0169 \\
\hline & women & 0.00010 & 0.0033 \\
\hline
\end{tabular}

Table 4: RSS for models fitted to 8 th best times

\begin{tabular}{|r|c|c|c|c|c|c|}
\hline & $\hat{A}$ & $\hat{B}$ & $\hat{C}$ & $\hat{\lambda}$ & $\operatorname{RSS}(\log (t))$ & $\operatorname{RSS}(v)$ \\
\hline men & $16.83(0.177)$ & $250^{*}$ & $4.66(0.028)$ & $-0.267^{+}$ & 0.00014 & 0.0053 \\
women & $15.80(0.064)$ & $250^{*}$ & $4.00(0.010)$ & $-0.267^{+}$ & 0.00002 & 0.0007 \\
\hline
\end{tabular}

Table 5: Parameters of model (6) (8th best times) 


\begin{tabular}{|r|rr|r|}
\hline Distance & Actual & Predicted & Residual \\
\hline men $1000 \mathrm{~m}$ & $2: 12.18$ & $2: 10.89$ & 1.29 \\
women $1000 \mathrm{~m}$ & $2: 29.33$ & $2: 25.43$ & 3.90 \\
men $3000 \mathrm{~m}$ & $7: 20.67$ & $7: 22.82$ & -2.15 \\
women $3000 \mathrm{~m}$ & $8: 06: 11$ & $8: 14.41$ & -8.30 \\
\hline men $5000 \mathrm{~m}$ & $12: 44.39$ & $12: 50.19$ & -5.80 \\
women $5000 \mathrm{~m}$ & $14: 36: 45$ & $14: 21.53$ & 14.92 \\
men $10000 \mathrm{~m}$ & $26: 38.08$ & $26: 58.73$ & -20.65 \\
women $10000 \mathrm{~m}$ & $29: 31.78$ & $30: 14.83$ & -43.05 \\
men marathon & $2: 06: 50.00$ & $2: 05: 05.00$ & $1: 45.00$ \\
women marathon & $2: 21: 06.00$ & $2: 20: 48.00$ & 18.00 \\
\hline
\end{tabular}

Table 6: Estimated record times, model (6)

\begin{tabular}{|c|c|c|c|c|c|c|c|}
\hline & & \multicolumn{3}{|c|}{ Chapman-Richards } & \multicolumn{3}{|c|}{ Anti-symmetric exponential } \\
\hline $\begin{array}{l}\text { Distance } \\
(\mathrm{m})\end{array}$ & $\begin{array}{l}\text { Record } \\
\text { (s), } 1996\end{array}$ & $\begin{array}{l}\text { Predicted } \\
\text { lower } \\
\text { bound }(\mathrm{s})\end{array}$ & $\begin{array}{l}\text { Difference } \\
\text { (s) }\end{array}$ & $\begin{array}{l}\% \text { of } \\
\text { limit }\end{array}$ & $\begin{array}{l}\text { Predicted } \\
\text { lower } \\
\text { bound }(\mathrm{s})\end{array}$ & $\begin{array}{l}\text { Difference } \\
\text { (s) }\end{array}$ & $\begin{array}{l}\% \text { of } \\
\text { limit }\end{array}$ \\
\hline 400 & 43.29 & 40.80 & 2.49 & 94.2 & 41.60 & 1.69 & 96.1 \\
\hline 800 & 101.73 & 94.85 & 6.88 & 93.2 & 97.00 & 4.73 & 95.4 \\
\hline 1500 & 207.37 & 192.69 & 14.68 & 92.9 & 197.50 & 9.87 & 95.2 \\
\hline 5000 & 764.39 & 715.18 & 49.21 & 93.6 & 735.00 & 29.39 & 96.1 \\
\hline 10000 & 1598.08 & 1499.63 & 98.45 & 93.8 & 1543.00 & 54.81 & 96.6 \\
\hline 42165 & 7610.00 & 6925.00 & 685.00 & 91.0 & 7143.00 & 467.00 & 93.9 \\
\hline
\end{tabular}

Table 7: Predicted record times, model (6) 


\section{Figures}

Figure 1: World records at end of 1996

Figure 2: World records on log scale

Figure 3: World record speeds

Figure 4: Speeds of 8th best times

Figure 5: Differences in speeds

Figure 6: Ratios of speeds

Figure 7: Variation in $a$, model (1)

Figure 8: Variation in $b$, model (1)

Figure 9: Variation in $A$, model (6)

Figure 10: Variation in $C$, model (6)
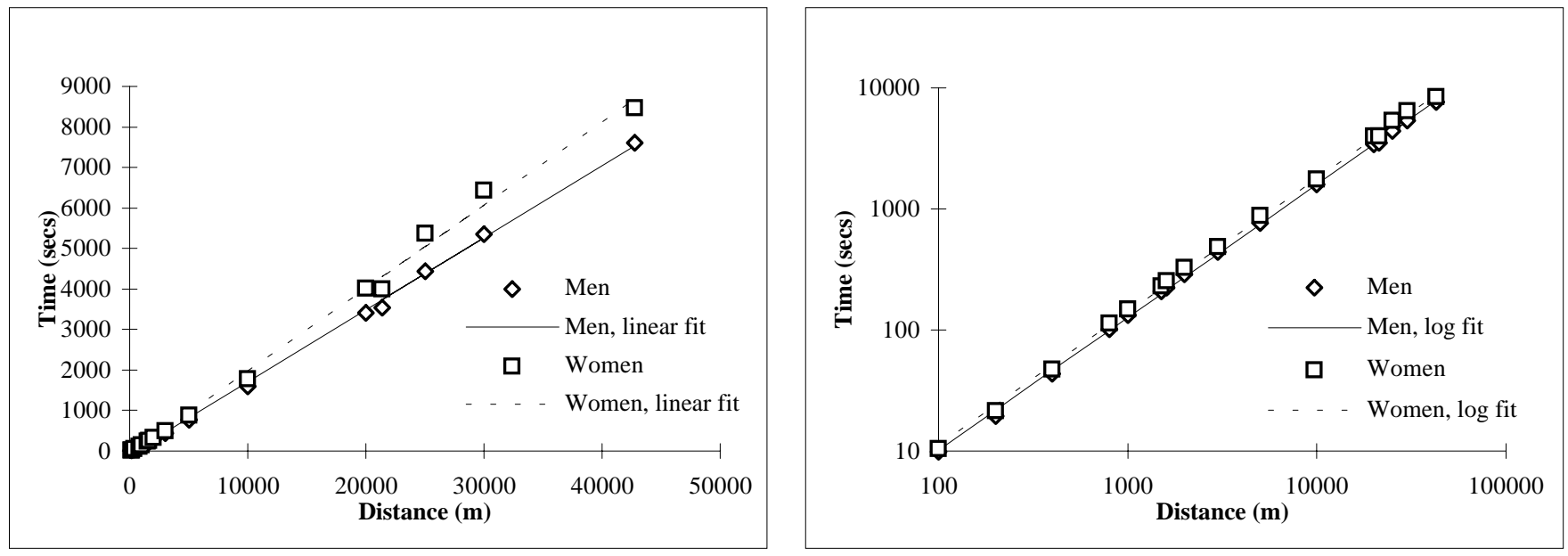

Figure 1: World records at end of 1996

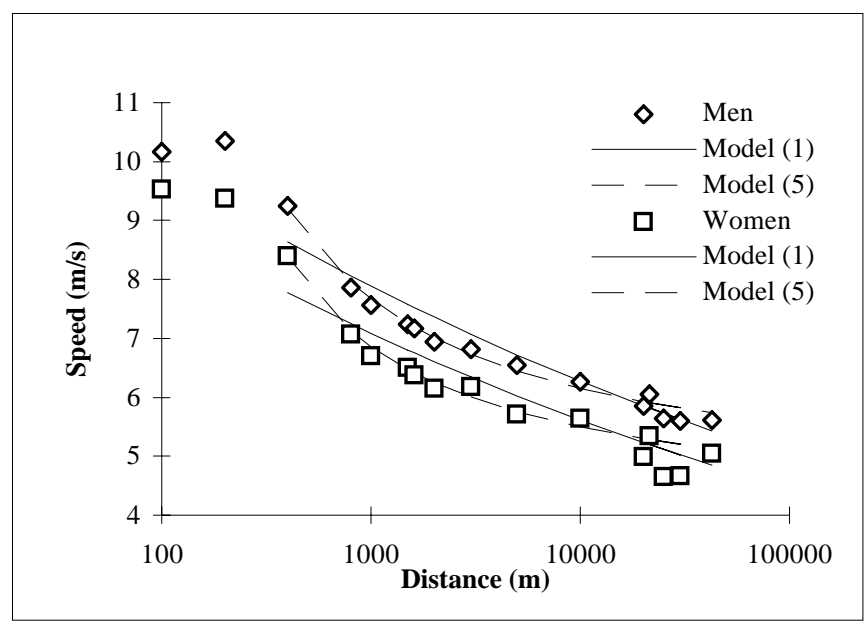

Figure 3: World record speeds

Figure 2: World records on log scale

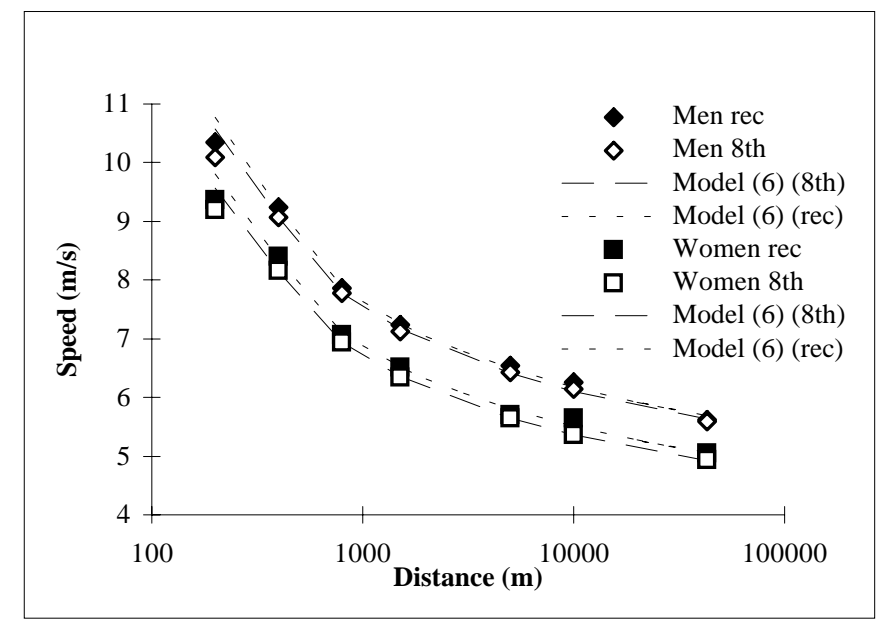

Figure 4: Speeds of 8 th best times 


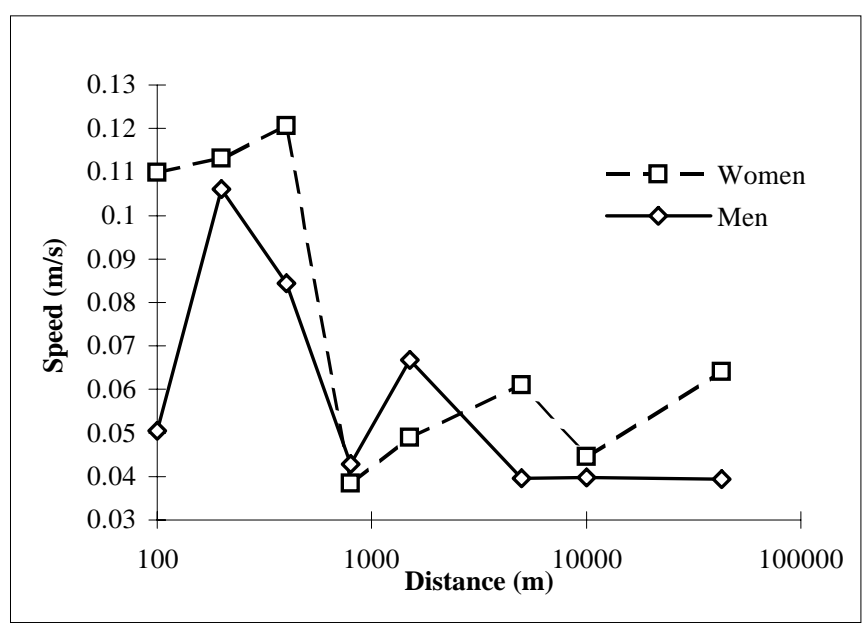

Figure 5: Differences in speeds

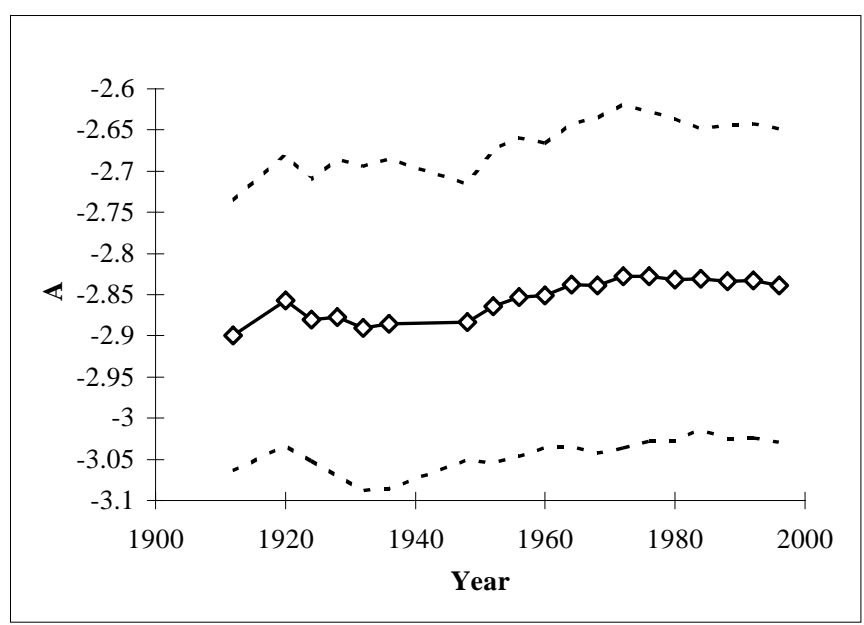

Figure 7: Variation in $a$, model (1)

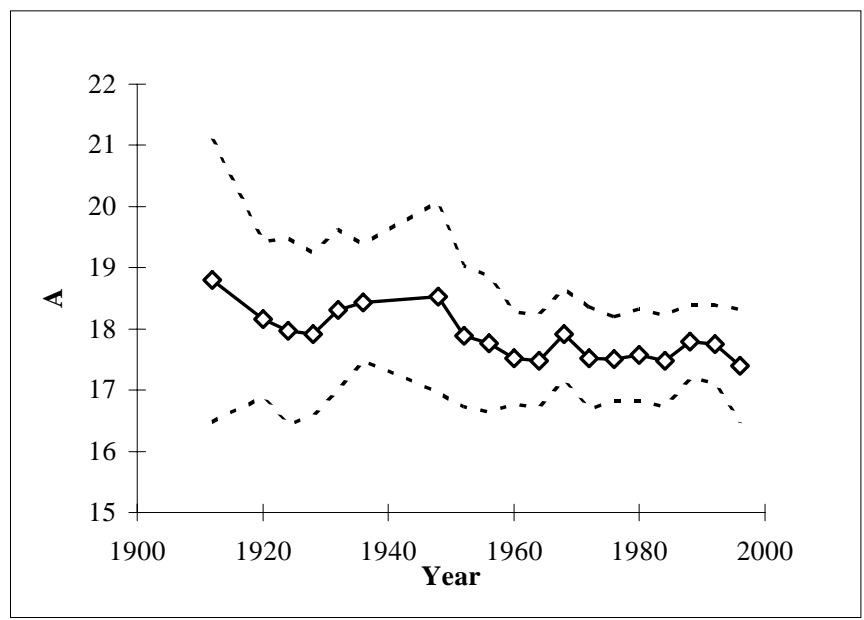

Figure 9: Variation in $A, \operatorname{model}(6)$

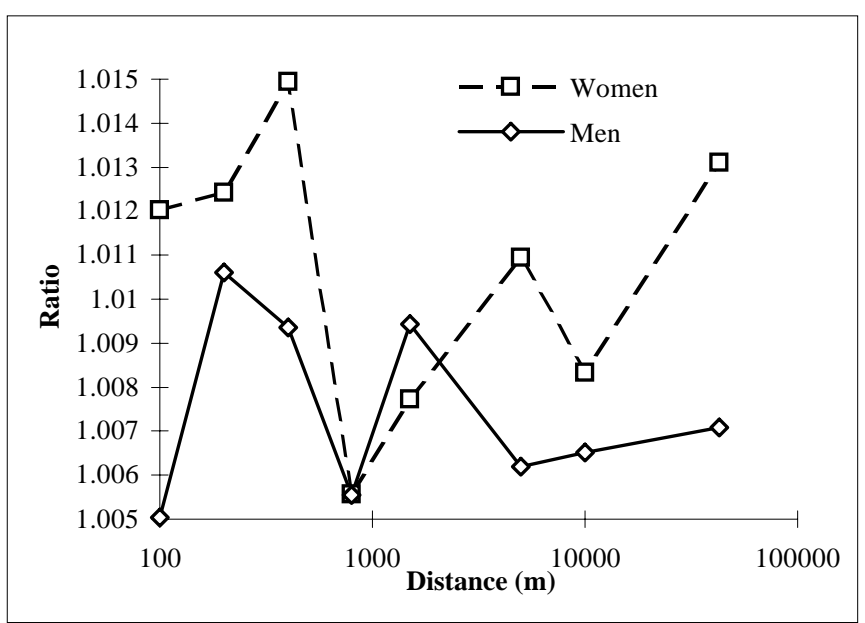

Figure 6: Ratios of speeds

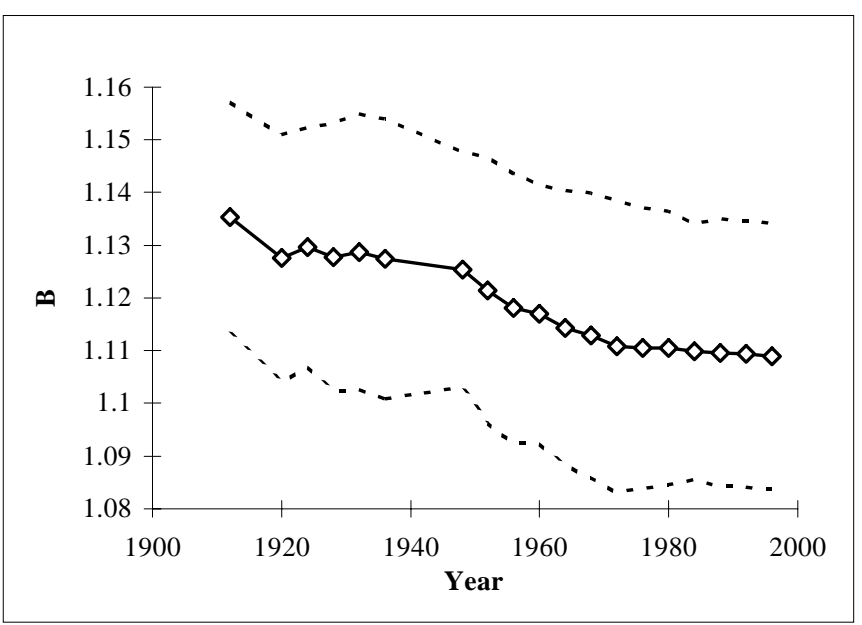

Figure 8: Variation in $b$, model (1)

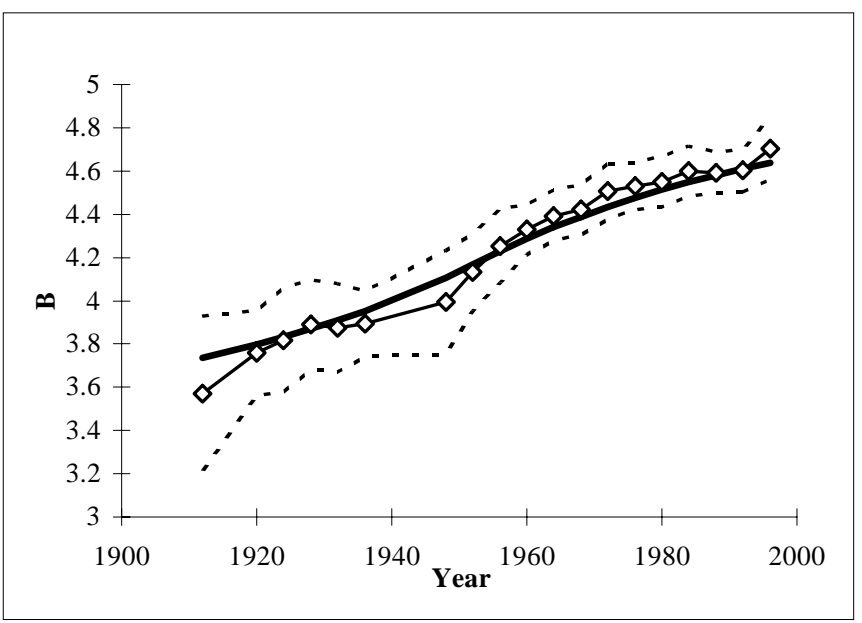

Figure 10: Variation in $C$, model (6) 